\title{
Pancreatic cystadenoma coexistent with incidental neuroendocrine tumor: a case report and literature review
}

Jingjin Wu ( $\sim$ 8017048@zju.edu.cn )

the fourth affiliated hospital, Zhejiang University School of Medicine

\section{Li Zhang}

the fourth affiliated hospital, Zhejiang University School of Medicine

Zhiyong Yu

the fourth affiliated hospital, Zhejiang University School of Medicine

\section{Case report}

Keywords: Pancreas, Cystadenoma, Serous, Neuroendocrine Tumors

Posted Date: March 5th, 2020

DOI: https://doi.org/10.21203/rs.3.rs-16175/v1

License: (c) (i) This work is licensed under a Creative Commons Attribution 4.0 International License.

Read Full License 


\section{Abstract}

Background Pancreatic cystadenoma coexistent with neuroendocrine tumor is a rare disease which is reported sporadically.

Case Presentation We herein present one such interesting case in a 67-year old man. There was only one mass detected preoperatively by different examinations. Endoscopic ultrasound-guided biopsy indicated a pancreatic neuroendocrine tumor. Therefore, a distal pancreatectomy was performed and the final histopathology revealed two separate masses in the pancreas, one was serious cystadenoma and the other neuroendocrine tumor.

Conclusions SCA coexistent with PNET is rarely found during clinical practice. The component of PNET may not be confirmed until the operation. Based on the recorded cases, the malignant potential, clinical characteristics, treatment, and prognosis are remaining further researches.

\section{Background}

Serous cystadenoma (SCA) of the pancreas is considered a rare pancreatic tumor with low malignancy and a comparatively good prognosis(1). Associated pancreatic neuroendocrine tumor (PNET) in SCA is furtherly rare. Heresbach et al reported the first case of an endocrine tumor arising in the pancreas with a separate serous cystadenoma(2). In the present study, we report an interesting case of SCA coexistent with PNET which was incidentally verified by fast-frozen pathology after resection of SCA. Besides, we reviewed the previous literatures that reported SCA associated with PNET.

\section{Case Presentation}

A 67-year old man had suffered from abdominal pain for nearly 20 years. He had undergone gastric repair 16 years ago and cholecystectomy 10 years ago. Four years ago, he was diagnosed as chronic atrophic gastritis with erosion. Enhanced CT found a cystic lesion in the pancreatic tail in the meantime, which was recommended regular observation due to the small diameter. After standardized treatment in the department of Digestion, gastric erosion was cured. However, he was still struggled against recurrent abdominal pain and admitted again. The physical examination and blood tests were unremarkable as well as the liver function, CA19-9 and colonoscopy. Enhanced CT found an irregular cystic lesion in the pancreatic tail which was considered as intraductal papillary mucinous neoplasm (IPMN) of branch duct type. In contract, enhanced MRI indicated that the cystic lesion measured $1.6 \mathrm{~cm}$ was firstly considered as serous cystadenoma. Then he had an endoscopic ultrasound (EUS) examination that indicated the presence of low echo lesion in the pancreatic body and tail as IPMN or PNET. Meanwhile, an EUS-guided biopsy was performed. Interestingly the biopsy considered highly differentiated neuroendocrine tumors. Although both a G1 PNET <2cm diameter and a small SCA are recommended observation, the patient firmly preferred radical resection due to the abdominal pain. Therefore, a laparoscopic operation resecting the distal pancreas was performed. A frozen section revealed two separate masses which were SCA and 
PNET respectively. Fortunately, the surgical margin was negative. Post-operative recovery was smooth and the patient was discharged 8 days after surgery.

Final histopathology was in line with frozen pathology. Two masses were seen in the section. The larger one was SCA measuring $1.2 \times 1.0 \mathrm{~cm}$ which was composed of the multilocular cystic cubic epithelium with no heteromorphism. The smaller was PNET (G1) which was located $5 \mathrm{~cm}$ away from the larger one near the resection margin of the pancreas. The diameter was $0.9 \mathrm{~cm}$. Microscopically, the tumor consisted of cells of relatively uniform size appearing as glandular, cord-like or solid growth. Some of the nuclei were filled with vacuoles. The nucleoli were visible and the capillary network was enriched. Immunohistochemistry showed positive neuron-specific enolase (NSE, +) and chromogranin A $(\mathrm{CgA},+)$.

\section{Discussion And Conclusions}

So far, pancreatic serous cystadenoma coexistent with neuroendocrine tumor is still a rare pancreatic disease. To date, cases were reported sporadically(2-5). Our case presented here is a 67-year old man. Although the ethnic origin is still unverified, it seems women have a higher incidence according to the literature $(3,6)$. In the case presented, the pancreatic double tumors were not discovered until the operation. There was only one mass detected preoperatively by different examinations with different results. What interesting is CT and EUS indicated that the pancreatic tumor was IPMN, while MRI was serous cystadenoma. Moreover, EUS biopsy considered highly differentiated neuroendocrine tumors. To our best knowledge, asymptomatic pancreatic cystic neoplasms with a size of less than $3 \mathrm{~cm}$ is recommended employing a conservative approach(7). According to the European Neuroendocrine Tumor Society, surgical resection is recommended for PNET $>2 \mathrm{~cm}(8)$. However, the patient had been enduring abdominal pain for a long time and he was firmly requested for operation. Besides, surgery is still the treatment of choice due to the significant malignant potential(9). Sharpe et al evaluated 380 PNETs in the National Cancer Data Base (NCDB). Five-year overall survival was $82.2 \%$ in the operation group and $34.3 \%$ in the observation group. They concluded that surgical resection provided an overall survival benefit for patients with small pancreatic neuroendocrine tumors(10). Finally, a distal pancreatectomy was performed. The postoperative histopathology discovered the real PNET incidentally which was not the lesion examined preoperatively. Similarly to the present case, Alasio et al reported a 78-year-old female case whose neuroendocrine tumor was discovered incidentally after complete resection of the serous cystadenoma(5). Consequently, the coexistence of two pancreatic tumors is easily misdiagnosed, so comprehensive evaluation is necessary before surgery.

The association between PNET and SCA remains unknown. One hypothesis is in light of the biphasic differentiation of the pancreatic ductal stem cells. Indeed, the biphasic differentiation from pancreatic ductal stem cells to either glandular epithelial or neuroendocrine cell has been found in many pancreatic tumors(11). While some tumors combine the epithelial and neuroendocrine features within the same cells, others show the epithelial and neuroendocrine cells developing concurrently within the same tumor(4). Further researches are still needed to figure out the association of coexistent PNET and SCA. 
Enhanced CT and MRI are commonly used during the diagnosis of pancreatic diseases. Using these image examinations, the tumor size and location can be easily verified as well as the anatomical relationship with surrounding organizations(12). EUS can effectively avoid the interference of intestinal gas, bone, and subcutaneous fat. Combined with high-frequency probe, a high-definition image is formed and the biopsy puncturing distance shortens. Furthermore, the immunohistochemical technique contributes to differential diagnosis on the molecular level. Kim Y. W used immunohistochemical studies dealing with PNET admixed with SCA firstly in the literature report(1). Neuron-specific enolase (NSE) and chromogranin $\mathrm{A}(\mathrm{CgA})$ are always positive in PNET. In the present study, we regret that somatostatinreceptor imaging, which contributed to exclude distant metastasis, was missed due to lack of necessary device.

To summarize, SCA coexistent with PNET is rarely found during clinical practice. The component of PNET may not be confirmed until operation. Based on the recorded cases, the malignant potential, clinical characteristics, treatment, and prognosis remain further researches.

\section{Abbreviations}

SCA: serous cystadenoma. PNET: pancreatic neuroendocrine tumor. IPMN: pancreatic neuroendocrine tumor. EUS: endoscopic ultrasound. NSE: neuron-specific enolase. CgA: chromogranin A.

\section{Declarations}

\section{Ethics approval and consent to participate}

This study protocol was reviewed and approved by the Ethics Committee of the Fourth Affiliated Hospital, Zhejiang University School of Medicine.

\section{Availability of data and material}

The datasets used during the current study are available from the corresponding author on a reasonable request.

\section{Consent for publication}

The patient described in the case presentation has given his written consent for his personal or clinical details along with any identifying images to be published in this study.

\section{Competing interests}


The authors declare that they have no competing interests.

\section{Funding}

Not applicable.

\section{Authors' contributions}

JW and LZ designed the study and drafted the manuscript. JW and ZY contributed to revising the manuscript.

\section{Acknowledgements}

Not applicable.

\section{References}

1. Kim YW, Park YK, Lee S, Park JH, Lee SM, Hong SW, et al. Pancreatic endocrine tumor admixed with a diffuse microcystic adenoma-a case report. Journal of Korean medical science. 1997; 12(5): 469-72.

2. Heresbach D, Robert I, Le Berre N, Raoul JL, Siproudhis L, Bretagne JF, et al. [Cystic tumors and endocrine tumor of the pancreas. An unusual association]. Gastroenterologie clinique et biologique. 1993; 17(12): 968-71.

3. Ustun MO, Tugyan N, Tunakan M. Coexistence of an endocrine tumour in a serous cystadenoma (microcystic adenoma) of the pancreas, an unusual association. Journal of clinical pathology. 2000; 53(10): 800-2.

4. Keel SB, Zukerberg L, Graeme-Cook F, Compton CC. A pancreatic endocrine tumor arising within a serous cystadenoma of the pancreas. The American journal of surgical pathology. 1996; 20(4): 4715 .

5. Alasio TM, Vine A, Sanchez MA, Dardik H. Pancreatic endocrine tumor coexistent with serous microcystic adenoma: report of a case and review of the literature. Annals of diagnostic pathology. 2005; 9(4): 234-8.

6. Blandamura S, Parenti A, Famengo B, Canesso A, Moschino P, Pasquali C, et al. Three cases of pancreatic serous cystadenoma and endocrine tumour. Journal of clinical pathology. 2007; 60(3): 278-82.

7. European Study Group on Cystic Tumours of the P. European evidence-based guidelines on pancreatic cystic neoplasms. Gut. 2018; 67(5): 789-804. 
8. Partelli S, Bartsch DK, Capdevila J, Chen J, Knigge U, Niederle B, et al. ENETS Consensus Guidelines for Standard of Care in Neuroendocrine Tumours: Surgery for Small Intestinal and Pancreatic Neuroendocrine Tumours. Neuroendocrinology. 2017; 105(3): 255-65.

9. Dong DH, Zhang XF, Poultsides G, Rocha F, Weber S, Fields R, et al. Impact of tumor size and nodal status on recurrence of nonfunctional pancreatic neuroendocrine tumors $</=2 \mathrm{~cm}$ after curative resection: A multi-institutional study of 392 cases. Journal of surgical oncology. 2019; 120(7): 10719.

10. Sharpe SM, In H, Winchester DJ, Talamonti MS, Baker MS. Surgical resection provides an overall survival benefit for patients with small pancreatic neuroendocrine tumors. Journal of gastrointestinal surgery : official journal of the Society for Surgery of the Alimentary Tract. 2015; 19(1): 117-23; discussion 23.

11. Tewari M, Patne S, Katiyar R, Biswas D, Shukla HS. Co-existence of Diffuse Serous Cystadenoma and Pancreatic Neuroendocrine Tumor. The Indian journal of surgery. 2017; 79(5): 455-7.

12. Agarwal N, Kumar S, Dass J, Arora VK, Rathi V. Diffuse pancreatic serous cystadenoma associated with neuroendocrine carcinoma: a case report and review of literature. JOP : Journal of the pancreas. 2009; 10(1): 55-8.

\section{Figures}



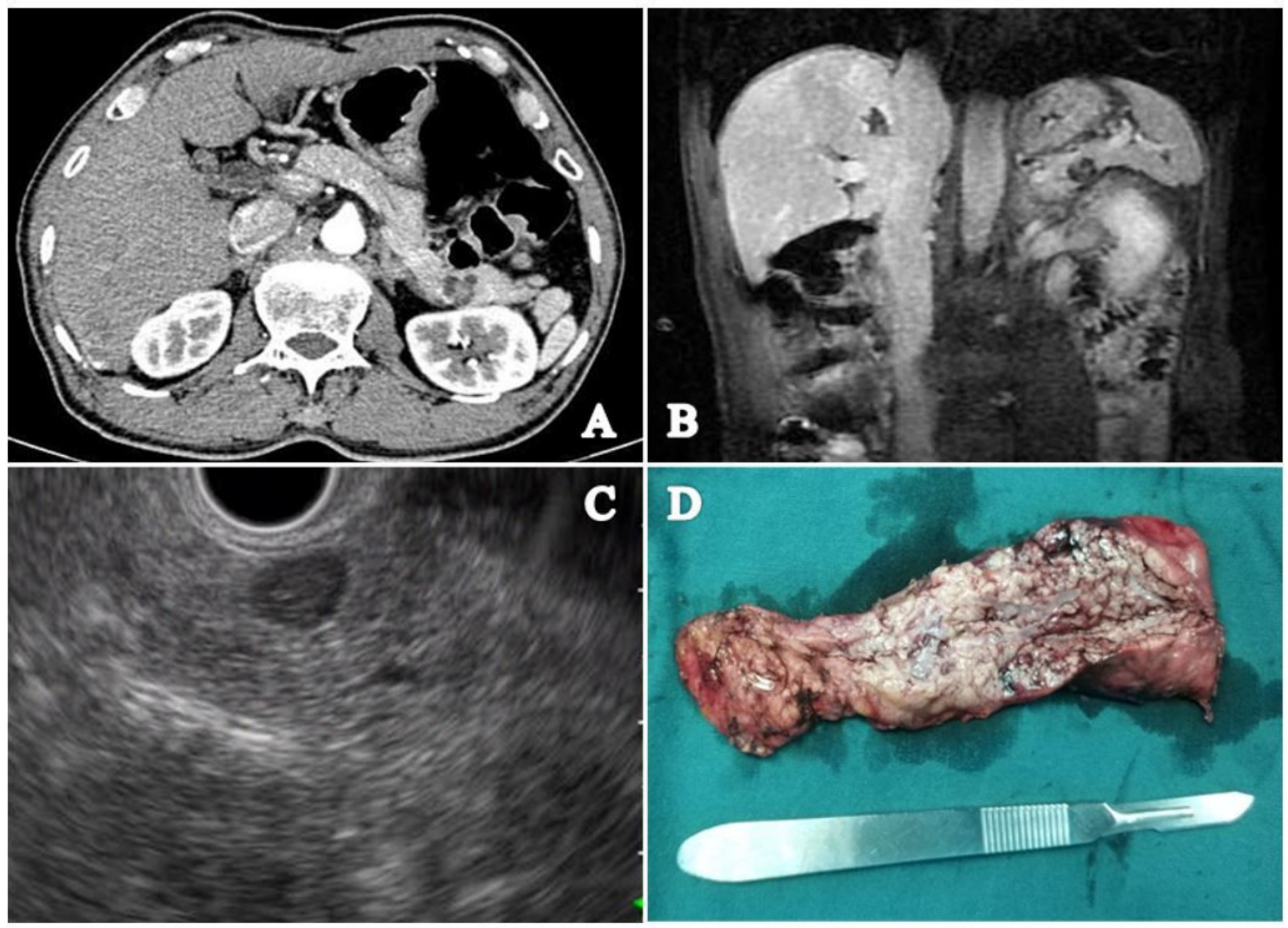

Figure 1

A The pancreatic tumor on enhanced CT. B The pancreatic tumor on enhanced MRI. C The pancreatic tumor on EUS. D The surgical specimen.

\section{Supplementary Files}

This is a list of supplementary files associated with this preprint. Click to download.

- CAREchecklist.pdf 